\title{
Research on the Development of Internet Insurance in China -Based on the exploration of the road of Huize insurance
}

\author{
Jing $\operatorname{Tian}^{1}$ \\ ${ }^{1}$ Glorious Sun School of Business and Management(GSSBM), Donghua University, Shanghai, 200051, China
}

\begin{abstract}
In 2020, Huize insurance came to the market in the United States, which will again push the Inter net insurance industry to the peak of social concern. As the one of three pillars of the financial industry, the Internet insurance industry is generated with the promotion of Internet finance. After experiencing a blowou t growth around 2015, the Internet insurance industry bypassed the monopoly of traditional insurance indust ry, At that time, big and small companies had their own characteristics. But as the policy tightens, Internet $\mathrm{i}$ nsurance suffers. With the impact of the epidemic in 2020, people's insurance awareness is constantly streng thened, and the Internet insurance industry will usher in a spring with its unique advantages. This paper com prehensively reviews the development of Internet insurance, analyzes the main problems of the Internet ind ustry, and put forward development recommendations based on Huize's business model, explore methods th e Internet industry in the social sense of responsibility, product design, and connect with the epidemic, comb ine with the government, to strengthen the construction of preferential and universal insurance, innovate the PPP mode, combine insurance technology to carry on the prospect.
\end{abstract}

\section{Introduction}

As a "stabilization tool" of the society and economy, the insurance industry shoulders the mission of promoting the economy and managing risks. With the continuous development of blockchain, big data and other Internet technologies, the Internet insurance market has begun to flourish and become an Internet finance market. As of 2015, China's Internet insurance premium income reached $\$ 76.836$ billion, experiencing a decline in early 2017. After that, the policy documents supporting the development of Internet finance have been issued intensively, adding wings to the development of Internet insurance. The COVID - 19 that happened in 2019 will have a profound impact on the transformation of the Internet insurance industry. This paper is based on Huize insurance, this paper is based on Smart Choice Insurance, which was listed in February 2020, and analyzes it from various perspectives such as business model, and then explores the future development path of Internet insurance companies. This will be of great significance to the reform of the insurance industry, the application of insurance technology, and the transformation and upgrading of the Internet insurance industry.

\section{Classification and product information of Internet insurance companies}

\subsection{Current situation of the insurance industry}

Internet insurance has been developing for 20 years since it sprouted in 2000, it has been developing for 20 years. Since 2017, China has become the second largest insurance market in the world. However, there is still a big gap between China's insurance depth and developed countries. China's insurance industry is still in the stage of development from "quantity" to "quality". As of 2018, the total premium income of China's insurance industry was 380.1662 billion yuan, a year-on-year increase of $3.92 \%$. Specifically, the main income of premiums is basically distributed in first- and second-tier cities, and the property and casualty income of the Tibet Autonomous Region is the lowest, only $1 \%$ of Jiangsu Province. This reflects the relatively solid development of my country's insurance industry, the lack of popularization of insurance awareness and social image, weaker awareness of insurance coverage in areas with lagging GDP, and low penetration rate of insurance industry. China's insurance development and popularization still have a long way to go. 


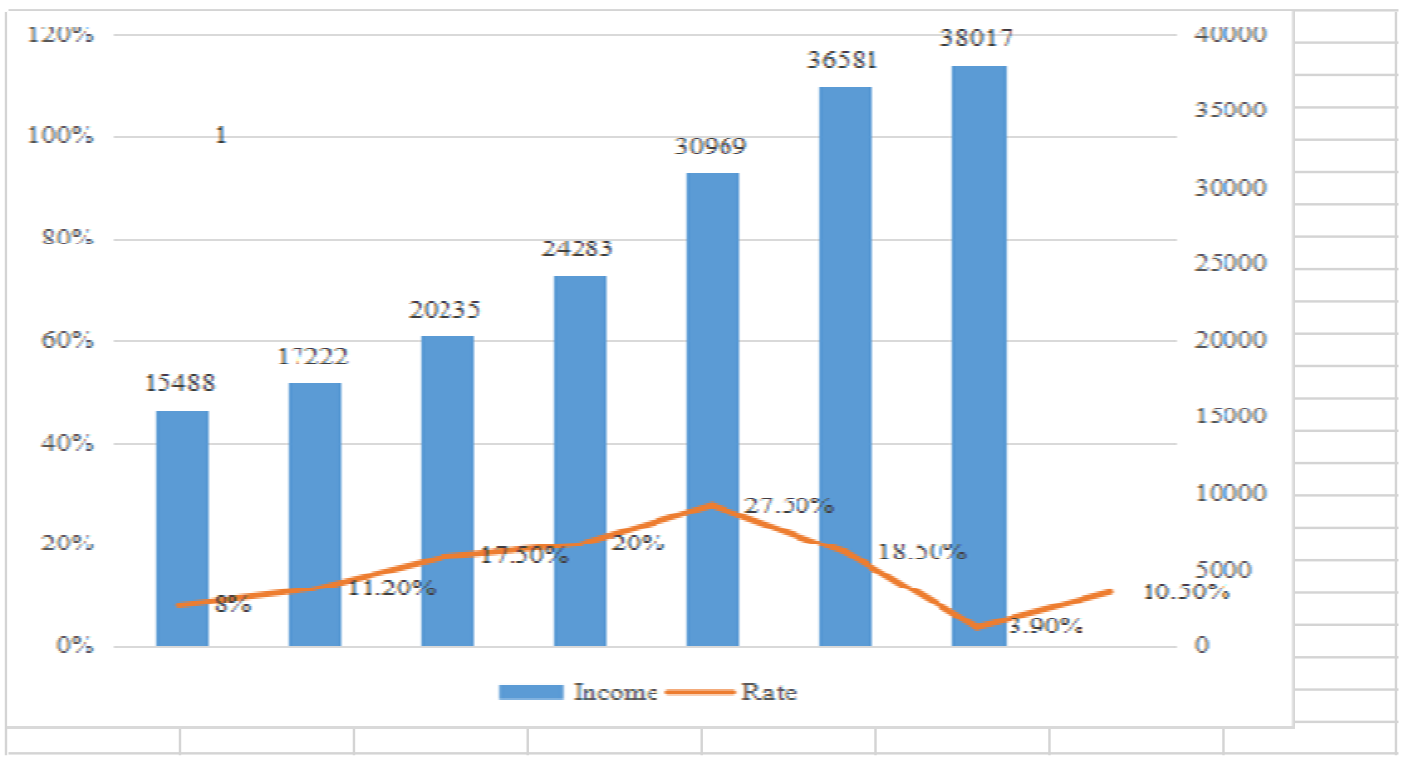

Fig1.2011-2019 China's original insurance premium income and its growth rate

\subsection{Current status of Internet insurance}

In December 1997, New China Life concluded the first Internet insurance policy in China. In 2006, an Internet third-party platform was launched, led by Huisheng.com. As of 2015, three insurance companies, Peace of Mind Insurance, Ease Insurance and Taikang Online, received internet insurance licenses, breaking the Zion Insurance a dominant situation. From 2010 to 2016, the state was encouraging to the Internet insurance industry. However, the Internet insurance industry suffered setbacks as integrity and regulatory issues emerged and national policy adjustments were made. The Internet insurance industry experienced short-term negative impacts under increasingly stringent policy laws. However, from a longterm perspective, the stringent policies and regulations have paved the way for the compliance and health of the Internet insurance industry.

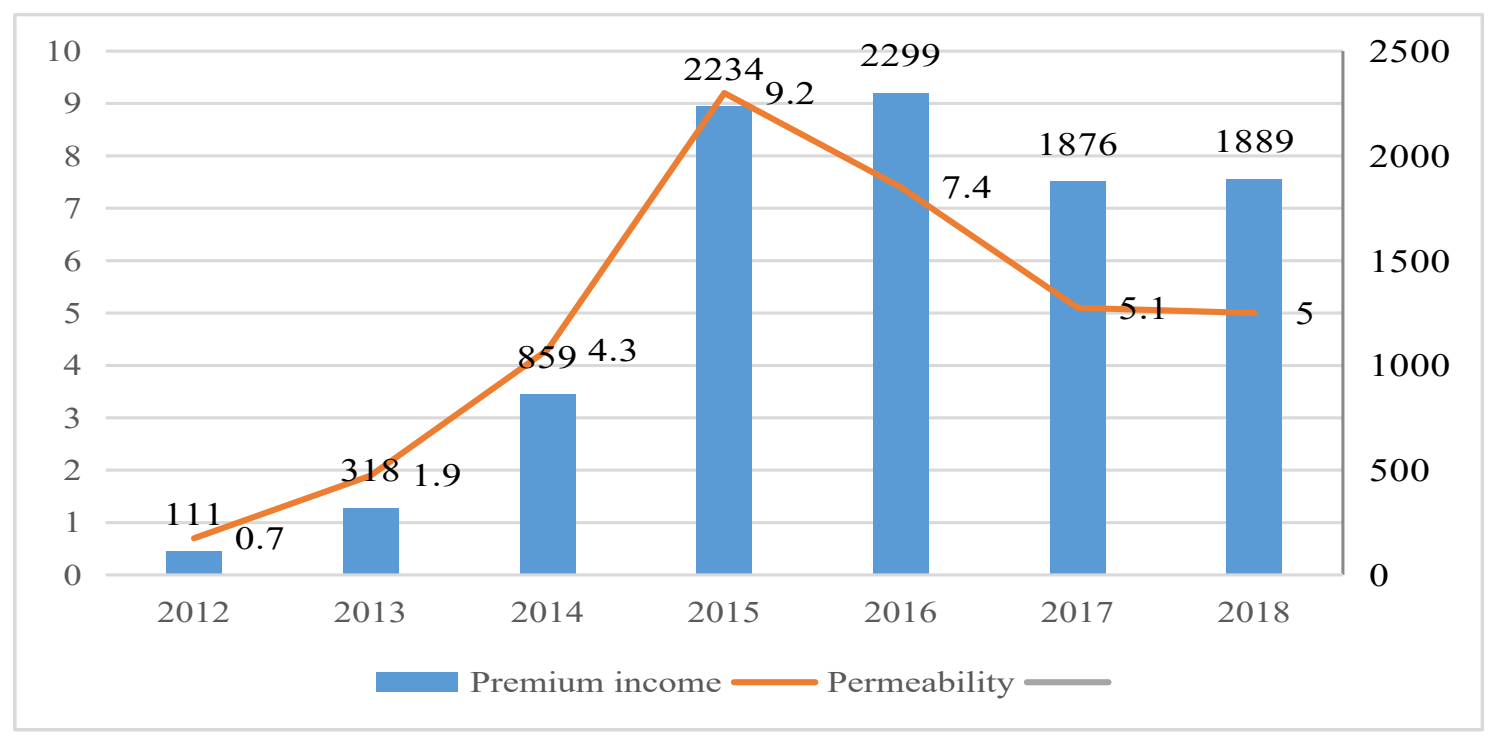

Fig2. China's Internet insurance revenue and penetration rate from 2012 to 2018

\subsection{Composition of Internet insurance companies}

At present, there are four types of Internet insurance companies in China. They are Internet third-party platforms, Internet channels for large insurance companies, Internet insurance companies with formal licenses, and small and medium-sized Internet insurance startups. In the early stage of development of the Internet insurance industry, third-party platforms were the mainstay. Then, the traditional Internet giants in China started their own online platforms and opened online sales channels, which provided the Internet giants with a wide range of services. The company has added a sales clientele and channels. With the development of technology such as blockchain technology and AI technology, insurance giants are beginning to focus on the internet insurance segment, with traditional insurance giants Improvements have been made to open the road to building Internet insurance as a new type of market. There is still a high concentration problem in the life insurance market, and the life insurance 
market share is still concentrated in the traditional banking online channel. Except for large platforms and insurance companies, Internet insurance is basically composed of small and medium-sized companies
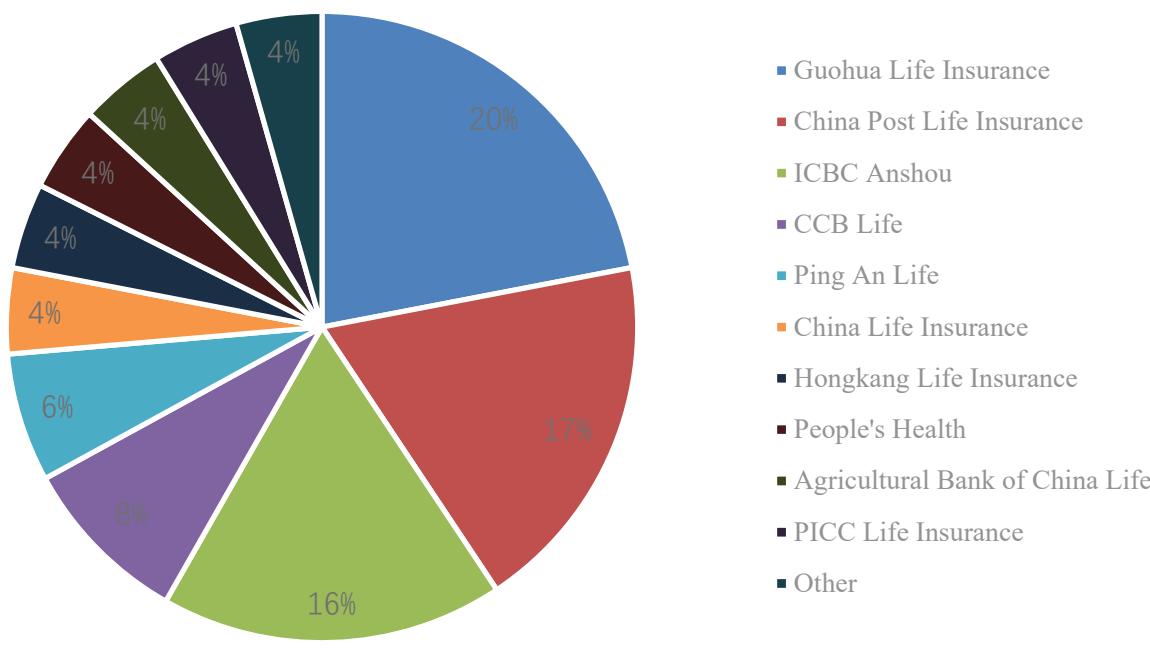

Fig3. Market share of internet personal insurance of various companies in 2019

billion in 2018. Zhang Ling et al. pointed out that Internet insurance is universal in nature and highly competitive. With the development of the new crown epidemic, more families began to pay attention to the protection of life insurance, life insurance will have a big breakthrough. In the initial period of internet insurance, auto insurance used to occupy the main position. With the deepening of the business car reform, the Internet car insurance impact, Internet insurance property insurance non-car insurance part of the growing day by day.

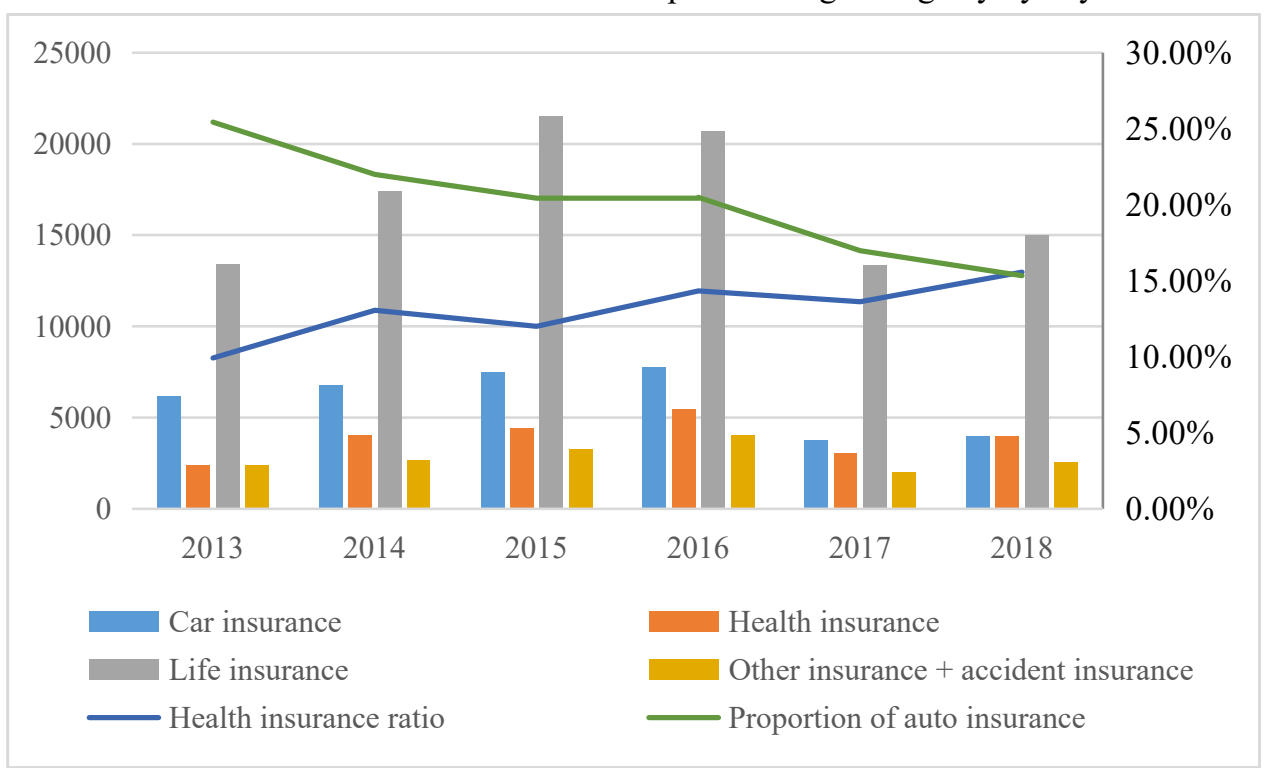

Fig4. China's insurance premium structure from 2013 to 2018

\section{Main problems facing Internet insurance}

\subsection{Serious product homogeneity}

At present, China's Internet insurance products are mostly concentrated in traditional personal insurance and property insurance. Most of these products are sold online based on traditional insurance products. The products are seriously homogenized and have a single form. They are greatly affected by macro factors such as policies. Therefore, it is urgent to change a single traditional product and develop a customized differentiated product that belongs to Internet insurance.

\subsection{Mistaking chicken ribs for innovation}

To solve the problem of single product, some companies have made attempts. For example, the sticker insurance 
and the Mid-Autumn Moon Insurance that received much attention recently. These insurance types are hung with the name of innovative products, and the essence is still traditional insurance. These "financial innovation products" deviate from financial ethics. Therefore, the true innovation of Internet products should be technological innovation from the perspective of promoting social progress, rather than launching some "gimmick" chicken taste products.

\subsection{It is difficult to build trust and awareness}

Unlike the physical traditional insurance industry, it is difficult to establish a trust mechanism in Internet insurance. For small and medium-sized Internet insurance companies, the limitations of scale and visibility make it difficult to gain the trust of customers, and thus they are unable to gain the trust of their customers. Of the four listed Internet insurance companies, all but the betterknown Zion Insurance were unable to open up awareness, resulting in Product development is hindered, which leads to a vicious circle that makes development difficult.

\subsection{Passive customer acquisition methods}

Compared to the traditional insurance industry, which is "proactive" and looking for potential customers, Internet insurance companies are in a more passive and browsing situation. This leads to a situation where customer traffic is not guaranteed. In the offline insurance business, each customer has a designated insurance agent to follow up with them and answer their questions, whereas online the customer initiates the Purchasing without an appointed agent can also lead to a lack of guidance when dealing with complex and specialized insurance provisions, reducing the customer's willingness to purchase.

\subsection{Lack of expertise}

For the Internet insurance industry, there is a need for professional insurance researchers and marketers. However, since the Internet insurance industry is in its nascent stage, it is relatively difficult to recruit good industry personnel. And unlike traditional insurance marketers, internet insurance professionals need to be proficient in data analysis, able to avoid risks, and innovative. Awareness and a deep understanding of the insurance industry and a forward-looking attitude. Therefore, how to attract talented professionals to the industry and how to train and deliver future talent is an issue that needs to be addressed.

\subsection{Product concentration on short-term insurance, resulting in price competition}

Currently, Internet insurance products are mostly focused on short-term insurance. This is due to various reasons such as lack of visibility and lack of customer trust in Internet insurance. However, the high homogeneity of short-term insurance has led to price competition among Internet insurance companies, triggering a vicious circle in the industry. The road to price competition leads to a dead end. Only when long-term insurance is introduced and the market is opened up, can companies escape from the cycle of price competition and find long-term development.

\section{Suggestions for development - Based on the practice of Huize insurance}

\subsection{Analysis of the Huize insurance}

When it comes to the Internet insurance platform, the most important one is Huize insurance, which was founded in 2006 and went public in the US after 14 years. Since its inception in 2006, Huize insurance has been listed on the US stock exchange for the past 14 years, and its long-term plan is worth exploring. Its main approaches are as follows.

The first is the transition from short-term insurance to long-term insurance, looking for profitable channels. As one of the first batch of Internet insurance companies in China to obtain Internet insurance licenses, Huize insurance's profit model is mainly to sell insurance products of cooperative insurance companies, in order to collect commissions. In 2019, based on the scale of premiums, Huize insurance has completed the shaping of subdivided fields, leading China's long-term life insurance and health insurance. 


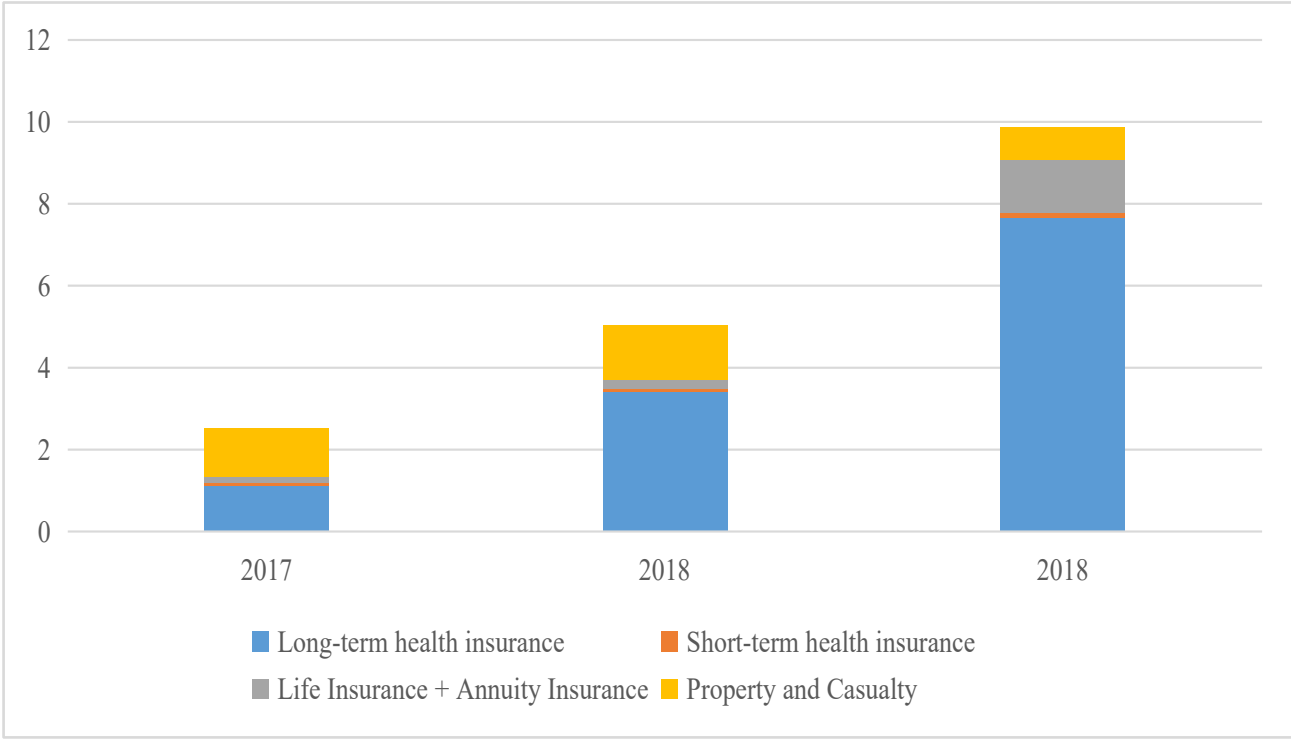

Fig5. Huize Insurance's income from various types of insurance

Second, it works closely with third-party platforms and multiple insurance companies to open marketing and product channels. In the sales channels of long-term life and health insurance of Wise Choice Insurance, indirect sales are a good way to avoid the trust of customers during the initial stage of Internet insurance. Huize insurance indirect sales and marketing channels mainly rely on media marketing. According to statistics, Huize insurance has reached cooperation with more than 2,000 self-media operators, to a certain extent, it helps to cultivate customers' insurance awareness and attract potential customers.

Thirdly, the target group of customers is young people with huge future customer potential. Huize insurance's special long-term insurance model provides it with customer retention. The younger generation accounts for a relatively large proportion of the insurance customer base, especially in its segment-leading long-term life and health insurance clients. Leaving valuable resources for future customer stickiness and market opening.

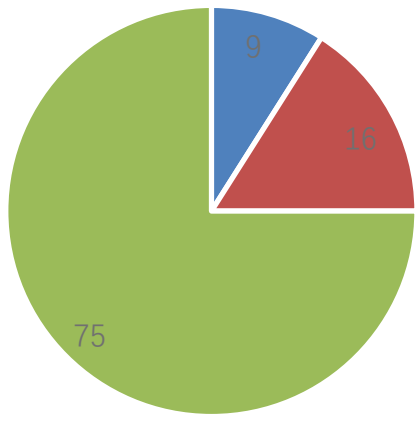

- Will not consider - Not sure - Accept

Fig6. Survey of netizens' acceptance of Internet insurance

Fourthly, we focus on customized services and create products by combining Internet technology. At present, Huize insurance not only promotes its products for B-tier insurance companies to solve the problem of customer acquisition, but also cooperates with upstream insurance companies to provide customized services. Open up explosive products. Personalized customization for each scenario of the product, and vigorously exploring customer needs, which is important for the future design of Internet insurance products. Lessons Learned.

\subsection{Suggestions for development based on the practice of Huize insurance}

One is to enrich the product connotation and improve the cost performance from the customer's point of view. For Internet insurance companies with a certain scale of operation, they need to jump out of the homogeneous products. From the perspective of users, they should combine big data and Internet technology to customize their own insurance products from the scene. From the successful experience of Huize insurance, we can see that internet insurance companies should focus on building "thousands of insurance products for thousands of people", combining big data and users. portrait and analyze users' risk preferences. At the same time, Internet insurance companies should improve product cost performance, accurately budget appropriate premiums with AI 
algorithm pricing model, and achieve rates Differentiation, making insurance coverage full coverage.

Secondly, we introduce talents and cultivate talents comprehensively. Strengthen the talent introduction and incentive system, vigorously develop the talent resource chain, provide rich safeguards for the introduction of talent, motivate and attract $\mathrm{AI}$ and big data and other technology talents, as well as insurance marketing, actuarial and other composite talents. At the same time, the Internet insurance company industry should pay attention to strengthening school-enterprise cooperation to cultivate future Internet insurance industry talents. It should learn from domestic and foreign talent training systems and improve the talent training system to save energy for the future development of the company and the industry.

Thirdly, we should cooperate with multiple channels and companies to achieve a win-win situation. Based on flow and product-oriented, we should strengthen cooperation with third-party platforms and self-media to accumulate flow through these channels. To locate the user positioning and conduct a wide range of insurance awareness to lead the society to understand the insurance industry and the Internet finance industry. Cultivate potential customers and increase insurance awareness of potential customers. Enterprises should pay attention to the exploration of potential customers and the cultivation of insurance awareness, and conduct insurance analysis and science popularization, not only to increase the public's insurance awareness, but also to Bring in potential customer resources and build the social image of the company itself.

\section{New coronary pneumonia-based outlook}

\subsection{Increased awareness of Internet insurance}

With the 2020 outbreak of the New Canopy epidemic, society was hit hard. The negative impact of Wuhan's closure of the city, the quarantine of residents' homes for two months, and the shutdown of industries have all led to an increase in social insurance awareness. At the same time, traditional insurers are accelerating their online business to promote the integration and transformation of online and offline business. According to China Securities Journal, the epidemic has pushed insurers to transform their business, accelerate the optimization of their business structure, and begin to focus on the full range of the Internet insurance sector, combined with insurance technology to accelerate the transformation and upgrading of the company. At the same time, the epidemic has driven insurance awareness, with residents checking the Internet for insurance-related knowledge and products during the quarantine period. Increased insurance awareness education and a foundation for opening up brand awareness for all internet insurance companies.

\subsection{Non-auto insurance continues to struggle, long-term insurance and health insurance have great prospects.}

The impact of the commercial car reform has impacted internet car insurance, as opposed to the rise of non-car insurance. The rising health and safety consciousness of the population and escalating security requirements have further contributed to the rise of personal health insurance The development. This year, in the case of the epidemic companies to stop for a longer period of time, the insurance industry's "good start" was affected, but life insurance has performed well. In February 2020 alone, the number of life insurance transactions under the impact of the epidemic has reached 95.41 million, an increase of 3.5 percent year-on-year. $1.22 \%$. The performance of this new coronary pneumonia is similar to that of 2003 SARS, and with reference to the trend of premium income during SARS, it can be inferred that the epidemic's Under the influence of health insurance development potential is huge. At the same time, crude dividends as well as the disappearance, the cost of relying on traffic alone is increasing. Occupy the medium and long-term insurance market, promote medium and long-term life insurance, health insurance development, in the Internet insurance industry has great significance.

\subsection{Take advantage of the nature of universal insurance, give full play to its advantages, and build "online insurance for big countries".}

Internet insurance has a natural value that is not limited by space and time, which gives it a universal nature. In the face of the epidemic, Internet insurance has demonstrated its enormous potential for providing economic security and safety to people in rural and remote areas of the country. Advantage. A series of documents issued by the $\mathrm{CBI}$ and the Development and Reform Commission (DRC) reflect the government's commitment to Internet commercial insurance in terms of social service Affirmation. There are already local governments and companies working together to form a PPP model to expand innovation and solve social problems. And Internet insurance, with its universal advantages such as cloud payments, wide coverage and low premiums, will contribute importantly to the economic recovery after the epidemic 5.4 Human-centered, integrated with insurance technology to achieve "quality" and "service" excellence.

\subsection{People-oriented, combined with insurance technology to achieve "quality" and "service" excellence}

Internet insurance companies should adhere to the peopleoriented, combined with the advantages of Internet insurance, to create cost-effective products. At present, there are phenomena such as charging high premiums for a large proportion of insurance in the market. These will affect the social image of the insurance industry and go against the principle of insurance protection. Under the impact of this epidemic, Internet insurance should take 
economic recovery and resident protection as the starting point, combining big data, AI and other technologies. Data to develop suitable products. At the same time, the Internet insurance industry can introduce AI customer service to explain insurance policies and complete expertise for customers.

\section{References}

1. Wei-Wei C, Liang-Rong S. Research on the Development Trend of Internet Insurance in China[J]. China Forestry Economics, 2017.

2. Jie-Yin H, Jin X U, Economics S O. Research on the Influence of Internet Insurance Development on the Efficiency of Insurance Companies-based on the DEA-Tobit Two Step Method[J]. East China Economic Management, 2019.

3. Hui-Xue W, Xin-Meng Z. Problems and Strategies of Integrity in Internet Insurance__ Based on the View of the Principle of Utmost Good Faith[J]. journal of changchun finance college, 2019.

4. Kittipaisalsilpa P. The Financial Game Changing: The Implications of Now-Trending Internet Finance in the Chinese Insurance Market[J]. Contemporary Social Science Journal of the Academy of Social Sciences, 2018, 27(3):165-181.

5. Qiming, Zhao, Dan,.A Study on the Relationship between the Development of Social Insurance Industry and Economic Growth in China[C]// 2018.

6. Kumar. D, Venkateswara K L, Siva P P . Internet of Thing (IOT) based Advance Insurance For Sensitive Food Products Transport Vehicle Using GPS Module[C]// 2019 International Conference on Vision Towards Emerging Trends in Communication and Networking (ViTECoN). 2019.

7. Hunt, Thomas, D. "The Internet of Buildings": Insurance of Cyber Risks for Commercial Real Estate[J]. Oklahoma Law Review, 2019.

8. Jiang Y, Luo Y, Xu X. Flood insurance in China: recommendations based on a comparative analysis of flood insurance in developed countries[J]. Environmental earth ences, 2019, 78(3):93.1-93.11.

9. Li L. Development and Innovation of Logistics Insurance in the Internet Age[J]. logistics engineering and management, 2019.

10. Lei Z, Yi-Qing X U, Lin S, et al. Research on the Effective Regulatory System of Internet Insurance Adapting to New Normal Status in China[J]. Journal of Finance and Economics Theory, 2018.

11. Lei Z, Yuhan X, Lu L, et al. Application of Blockchain in Promoting High-quality Development of Internet Insurance[J]. Exploration of Financial Theory, 2018.

12. Baorong Y U, Jin Y, Xifei G, et al. Development process, business modes and macrocally influencing factors for Internet-based health service in China[J]. journal of shandong university (health sciences), 2019. 